Case Report

\title{
Extensive Bone Lesions and Skin Lesions Revealing an Acute Transformation of an Extramedullary Chronic Myeloid Leukemia
}

\author{
Bienvenu Houssou ${ }^{1,}$, , Mouna Lamchahab ${ }^{1}$, Halima Hadri ${ }^{1}$, Boubker Messoudi ${ }^{2}$, \\ Abdeljabbar Messoudi ${ }^{2}$, Asma Quessar ${ }^{1}$ \\ ${ }^{1}$ Department of Clinical Hematology and Pediatric Oncology, Hospital August 20th 1953, University Hassan II, Casablanca, Morocco \\ ${ }^{2}$ Department of Trauma and Orthopedic of the Hospital Ibn Rochd, University Hassan II, Casablanca, Morocco
}

\section{Email address:}

houbien85@yahoo.fr (B. Houssou), mlamchahab@hotmail.fr (M. Lamchahab), hdrhalima@gmail.com (H. Hadri),

dr.messoudi.boobker@gmail.com (B. Messoudi),Abdljbbar@yahoo.fr (A. Messoudi), quessar.a@gmail.com (A. Quessar)

${ }^{*}$ Corresponding author

\section{To cite this article:}

Bienvenu Houssou, Mouna Lamchahab, Halima Hadri, Boubker Messoudi, Abdeljabbar Messoudi, Asma Quessar. Extensive Bone Lesions and Skin Lesions Revealing an Acute Transformation of an Extramedullary Chronic Myeloid Leukemia. Cancer Research Journal.

Vol. 6, No. 2, 2018, pp. 70-73. doi: 10.11648/j.crj.20180602.15

Received: March 13, 2018; Accepted: March 29, 2018; Published: May 5, 2018

\begin{abstract}
Extramedullary blast transformation as the initial presentation of Chronic Myeloid Leukemia with bone marrow remaining in the chronic phase is an unusual event. We report the case of a 30-year-old with chronic CML at the medullary level and an acute transformation revealed by extensive bone lyses and skin lesions. A 30-year-old man had cutaneous nodules, functional impotence and paresis. Radiological investigations revealed osteolytic lesions of the right humeral head, right clavicle, ribs, C5 and C7 vertebrae with medullary compression. Anatomo-pathological study and immunohistochemistry of nodules and osteolytic lesions resulted in granulocytic sarcoma with CD10 +, CD34 +, CD13 +, CD33 +, CD117 and MPO positive. The myelogram was in favor of chronic phase CML. He was put on imatinib, the chemotherapy could not be started because his condition was impaired and he dies by metabolic disorder. The acute transformation of chronic phase CML into extra marrow remains unusual. The care is a real challenge hence the need for consensual recommendations.
\end{abstract}

Keywords: Acute Transformation, Chronic Myeloid Leukemia, Bones lesions, Skin Lesions

\section{Introduction}

Chronic myeloid leukemia (CML) is a myeloproliferative neoplasms characterized by the presence of the Philadelphia chromosome generated by the reciprocal translocation $\mathrm{t}(9 ; 22)$ (q34; q11). CML is usually diagnosed in the chronic phase. The majority of extramedullary involvement occurs in association with peripheral and / or medullary blastosis; in rare cases, it may be manifested as a soft tissue leukemic infiltrate (chloroma, myeloblastoma or myeloid sarcoma) of different sites, such as the lymph nodes, the synovium or the bone $[1,2]$. Extramedullary blast transformation as the initial presentation of CML with the bone marrow remaining in the chronic phase is an unusual event [3].
We report the case of a 30-year-old with chronic CML at the medullary level and an acute transformation revealed by extensive bone lyses and skin lesions.

\section{Observation}

A 30 year-old man was admitted in consultation for spontaneous pains of the right shoulder with the notion of loss of consciousness associated with a progressive asthenia over the days. During the physical examination, the patient was in a fairly good general condition, PS (WHO): 2, conscious, stable hemodynamically and on respiratory terms, apyretic, clean tongue, caries of teeth $15,16,23,24$ and 35; swelling of the right shoulder with edema covering the entire right thoracic 
limb, presence of nodules covered with scales on the right shoulder and the right arm. He had a paresis. Para-clinical examinations note on the radiograph of the right arm: osteolytic lesions of the upper $1 / 3$ of the right humerus, englobing the humeral head (Figure 1). MRI of the right arm showed a voluminous epiphyso-metaphyso-diaphyseal right humeral tumoral process with invasion of the scapula, the clavicle, soft tissues, and skip metastases, lymphadenopathies with probably a costal damage suggestive of a prior lytic osteosarcoma or Ewing sarcoma. Medullary MRI revealed lytic lesions of $\mathrm{C} 5$ and $\mathrm{C} 7$, medullary compression of $\mathrm{C} 5$ to $\mathrm{C} 7$ (Figure 2). The bone scintigraphy revealed multiple osteolytic foci which lead us to evoke primarily rapidly evolving and aggressive bone metastases. A biopsy of the bone lesions of the right humeral head was consistent with a plasmocytoma without eliminating other round-cell malignancy, and at the immunohistochemistry, the tumor cells expressed CD45 (clone 2B11), and the MPO, in a vague to focal manner, expressed CD10, CD34, CD13, CD33 and CD117 were diffusely positive, CD20, CD3, Tdt, CD99 were negative and brought us to the conclusion that it's a granulocytic sarcoma. The biopsy and the immunohistochemistry of cutaneous nodules found a cutaneous localization of an acute myeloid leukemia. The myelogram of a marrow which was very rich in cells such as megakaryocytes revealed a hyperplasia of the myeloid lineage with eosinophilia, and an intense basophilia without any sign of an acute transformation and concluded a chronic myeloid leukemia in its chronic phase. The medullary karyotype revealed on 22 mitoses 4 cell clones: an initial clone with $\mathrm{t}(9 ; 22)$, a Philadelphia chromosome positive, a first sub-clone with trisomy 17 and trisomy 19, a second sub-clone with a trisomy 8 , a mitosis with a double chromosome Philadelphia. The BCR-ABL rearrangement was positive in $65 \%$ of the cells analyzed. The electrophoresis of serum proteins was normal. The hemogram revealed: $\mathrm{Hb}: 13.4 \mathrm{~g} / \mathrm{dl}$, MCV: 88\%, WBC: 15.4 G / L, PNN: 8.9 G / L, Lymphocytes: 5 G / L, Basophils: 0.6 G/L, Platelets: 1680 G / L. In addition, the abdominal and cardiac ultrasound, and the electrocardiogram were normal.

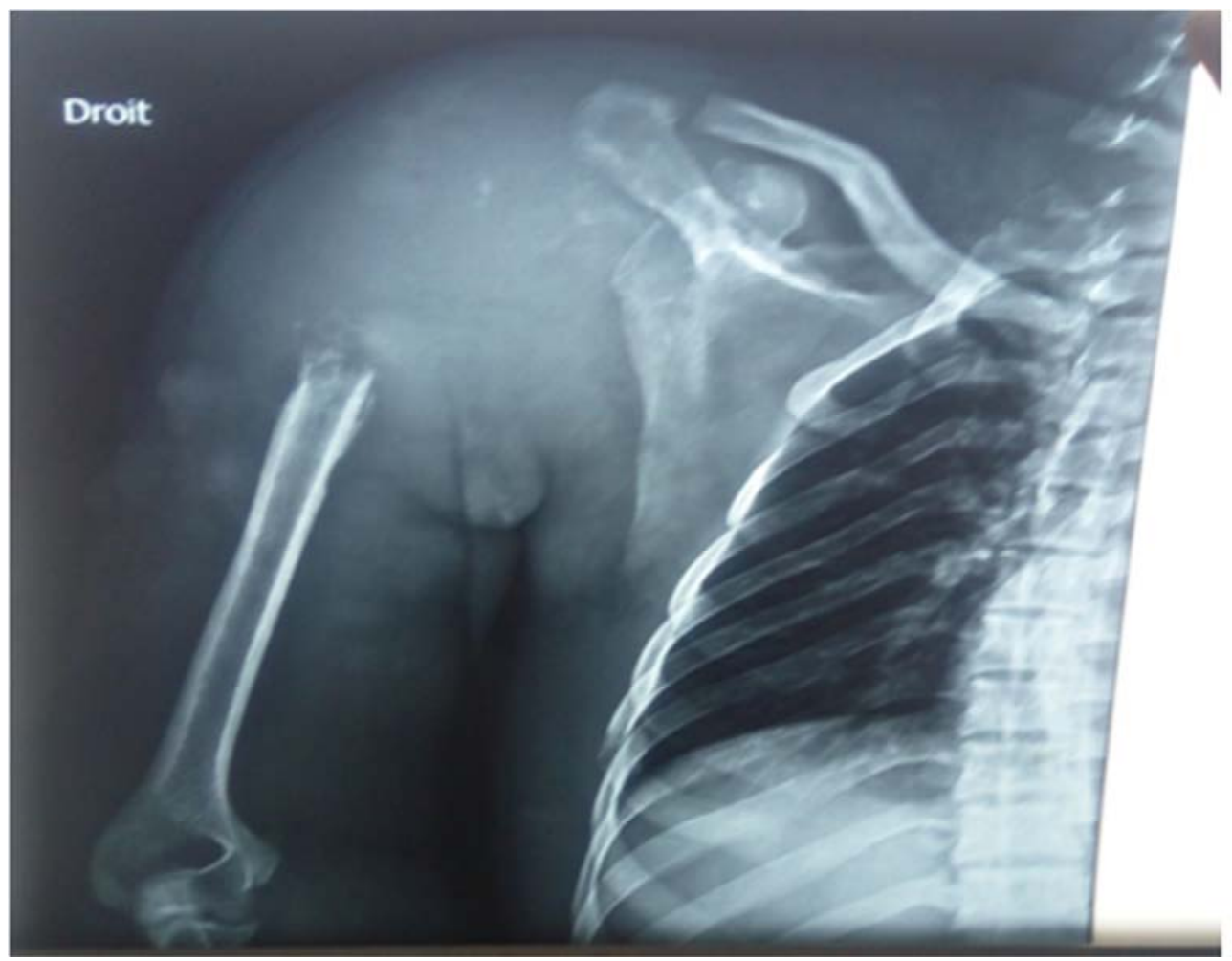

Figure 1. Total lysis of the right humeral head with demineralization of the bone.

Cumulatively, this is a young man of 30 years, presenting an acute extra medullar transformation of a CML revealed by extensive bone lesions and a cutaneous localization.

The file was discussed in a multidisciplinary consensual meeting between hematologists, radiologists, pathologists, radiotherapists and neurosurgeons; they decided not to operate so as not to aggravate his clinical condition, to do radiotherapy on the cervical spine, to start imatinib $600 \mathrm{mg} /$ day and to do as soon as possible, chemotherapy and allograft.

He was hospitalized, put on Imatinib; he received 20 Grays of radiotherapy at the rate of 2 grays per day for 10 days. He presented a post-radiation mucositis that had evolved well under treatment. During his hospitalization, his general condition deteriorated as days passed by, he had presented a pancytopenia which had required the stop of the Imatinib and the launch of a treatment by growth factor; metabolic disorders: severe hyponatremia, hypocalcemia which had been corrected. He had necrosis of the penis requiring the placement of a cystostomy and he died on the 52nd day of hospitalization due to neurological distress. 


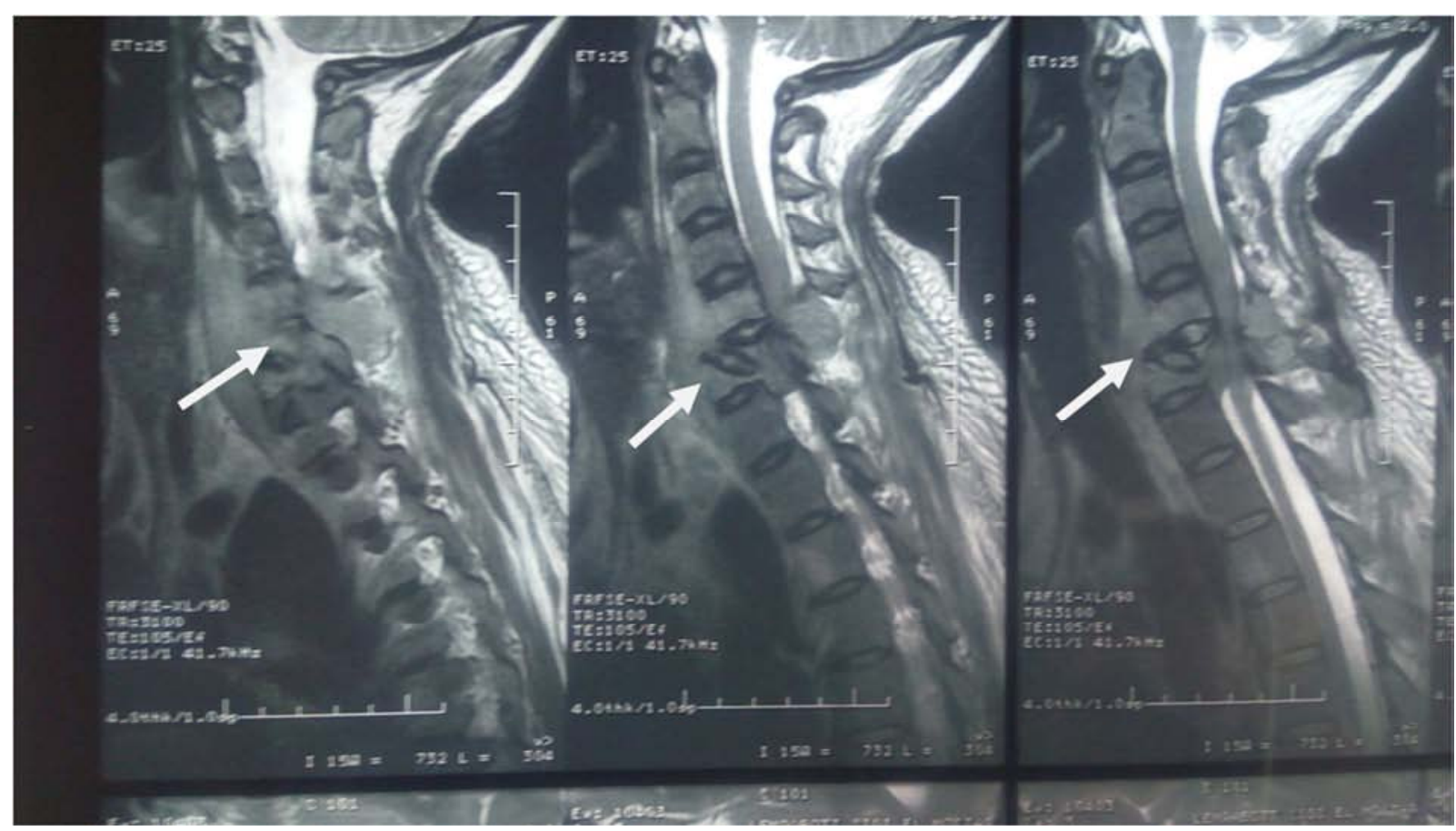

Figure 2. Strongly lesional spine of C5 to C7 with complete settling, recoil of the posterior wall and medullary compression.

\section{Discussion}

The acute extramedullary transformation of CML is defined as the infiltration of leukemia blasts into regions other than the bone marrow, which has been reported in only 4 to $16 \%$ of cases of CML [4].

Several authors have reported that the bone is a predilection site for acute transformation of CML [1, 2, 5-7] but none of them reported an acute transformation with the bone marrow remaining in the chronic phase. Our patient had presented at the stage of diagnosis a CML in its chronic phase on the bone marrow and an acute extramedullary transformation revealed by important bone lesions, which gave rise to the suspicion of, taking into account the patient's age (30 years), the metaphyso-diaphysaire location on the right humerus and the rapid evolution with invasion of the scapula, the clavicle, the coast and metastatic skips, an Ewing's sarcoma or osteosarcoma. Research over the past 50 years in the medline database found no reported cases of chronic bone marrow CML and an acute extramedullary transformation of the bones and at the same time on the skin.

To our patient we proposed the tyrosine kinase inhibitor (TKI), Imatinib because it is the most accessible. Dasatinib could be proposed because it is a second-generation TKI with the oncogene BCR-ABL inhibitory activity 325 -fold greater than imatinib in vitro [8] and it has been proven that it is capable of penetrating the blood-brain barrier [9].

While $\mathrm{Yu} \mathrm{HH}$ et al reported that extramedullary blasts crisis have traditionally been linked to poor survival [10], the difficulty of managing our complicated patient is related firstly to the extent of the lesions and secondly to the lack of clear, concise strategies recommended for the management of such a patient.

\section{Conclusion}

An acute transformation of chronic phase CML into extra marrow remains unusual. The care is a real challenge hence the need for consensual recommendations.

\section{Compliance with Ethical Standards}

This study did not receive any funding.

\section{Ethical Approval}

All procedures performed in studies involving human participants were in accordance with the ethical standards of the institutional and/or national research committee and with the 1964 Helsinki declaration and its later amendments or comparable ethical standards.

\section{References}

[1] Jin GN, Zou P, Chen WX, Ding ZY, Zhou H. Fluorescent in situ hybridization diagnosis of extramedullary nodal blast crisis. Diagn Cytopathol. 2013; 41 (4):253-256.

[2] Shune L, Cayci Z, Rogosheske J, Brunstein C, Ustun C. Extramedullary blastic crisis in abdominal lymph nodes in a patient with chronic myelogenous leukemia on imatinib. Leuk Res. 2012; 36 (6):e131-e132.

[3] Gao X, Li J, Wang L, Lin J, Jin H, Xu Y, Wang N, et al. Bilineal Extramedullary Blast Crisis as an Initial Presentation of Chronic Myeloid Leukemia: A Case Report and Literature Review. Am J Case Rep. 2016: 27; 17:793-798. 
[4] Levy RA, Mardones MA, Burch MM, Krause JR. Myeloid sarcoma as the presenting symptom of chronic myelogenous leukemia blast crisis. Proc (Bayl Univ Med Cent) 2014; 27 (3):246-49.

[5] Kantarjan HM, Keating MJ, Talpaz M. Chronic myelogenous leukemia in blast crisis: analysis of 242 patients. Am J Med. $1987 ; 83: 445-454$.

[6] Canellos G. Clinical characteristics of the blast phase of chronic granulocytic leukemia. Hematol Oncol Clin North Am. 1990; 4:359-376.

[7] Specchia G, Palumbo G, Pastore D, Mininni D, Mestice A, Liso V. Extramedullary blast crises in chronic myeloid leukemia. Leuk Res. 1996; 20:905-908.
[8] O'Hare T, Walters DK, Stoffregen EP, Jia T, Manley PW, Mestan J, et al. In vitro activity of Bcr-Abl inhibitors AMN107 and BMS-354825 against clinically relevant imatinib-resistant Abl kinase domain mutants. Cancer Res. 2005; 65 (11):45004505 .

[9] Porkka K, Koskenvesa P, Lundan T, Rimpilainen J, Mustjoki S, Smykla R, et al. Dasatinib crosses the blood-brain barrier and is an efficient the therapy for central nervous system Philadelphia chromosome-positive leukemia. Blood. 2008; 112 (4):10051012 .

[10] Yu HH, Lu MY, Lin DT, Lin KH, Tang JL, Jou ST. Pathological fracture as a manifestation of extramedullary blastic crisis in chronic myelogenous leukemia: report of one case. Acta Paediatr Taiwan. 2006; 47 (3):150-154. 